\title{
Risk prediction model for epithelial ovarian cancer using molecular markers and clinical characteristics
}

\author{
Meiying Zhang ${ }^{1,2}$, Guanglei Zhuang ${ }^{3}$, Xiangjun Sun ${ }^{1,2}$, Yanying Shen ${ }^{4}$, Aimin Zhao ${ }^{1,2}$ and Wen Di ${ }^{1,2^{*}}$
}

\begin{abstract}
Background: A high-quality risk prediction model is urgently needed for the clinical management of ovarian cancer. However most existing models are solely based on clinical parameters, and molecular classifications in recent reports are still being debated. This study aimed to establish a risk prediction model by using both clinicopathological and molecular factors (the synthetic model) for epithelial ovarian cancer.

Methods: A retrospective cohort study was conducted in epithelial ovarian cancer patients $(n=161)$ treated with primary debulking surgery and adjuvant chemotherapy. The expression level of 15 selected molecular markers were measured using immunohistochemistry. A risk model was developed using COX regression analysis with overall survival as the primary outcome. A simplified scoring system for each prognostic factor was based on its coefficient. Independent validation $(n=40)$ was conducted to evaluate the performance of the model.

Results: A total of 10 out of 15 molecular markers were significantly associated with clinical characteristics and overall survival. The synthetic model performed better than the clinicopathological risk model or the molecular risk model alone, as assessed by analysis of the receiver-operating characteristics curve area and the Youden index. The synthetic model included parity (>3), peritoneal metastasis, stage, tumor type, residual disease, and expression of human epidermal growth factor receptor 2 (HER2), epidermal growth factor receptor (EGFR), breast cancer 1 (BRCA1), murine sarcoma viral oncogene homolog B (BRAF) and Kirsten rat sarcoma viral oncogene homolog (KRAS).
\end{abstract}

Conclusions: Our synthetic risk model may more accurately predict survival of epithelial ovarian cancer patients than current models.

Keywords: Epithelial ovarian cancer, Risk prediction model, Molecular classification, Overall survival

\section{Background}

Risk prediction in patients with intractable disease is one of the major challenges for both clinic treatment and basic research, which tailors risk assessment of outcome based on the individual's clinical, epidemiological and molecular factors [1]. By means of the risk prediction model, disease can be more efficiently monitored and precisely treated. Ovarian cancer is the most lethal gynaecologic malignancy.

\footnotetext{
*Correspondence: diwen163@163.com

'Department of Obstetrics and Gynecology, Ren Ji Hospital, School of Medicine, Shanghai Jiao Tong University, 160 Pujian Road, Shanghai 200127, China

${ }^{2}$ Shanghai Key Laboratory of Gynecologic Oncology, Ren Ji Hospital, School of Medicine, Shanghai Jiao Tong University, 160 Pujian Road, Shanghai 200127, China

Full list of author information is available at the end of the article
}

Despite improvements in diagnostic methods, surgical technologies and chemotherapeutic agents over the past few years, the prognosis is still poor [2-5]. Therefore, the development of a high-quality risk prediction model for ovarian cancer to guide personalized therapy is a primary research focus in the field.

In 1989, Van Houwelingen first reported a prognostic index (PI) for ovarian cancer [6]. Since then, a series of studies reported that the prognostic model, which is based on clinical characteristics including advanced age, higher stage and grade of tumor, presence of ascites, poorer performance status and residual disease $(>1 \mathrm{~cm})$, was able to stratify patients with poor survival [7-9]. However, ovarian cancer patients with similar clinical characteristics also exhibit difference in prognosis, which 
may be due to high molecular heterogeneity of tumor and/or different molecular genetics [10, 11]. It has been reported that expression or status of certain molecular markers, such as tumor protein 53 (TP53), epidermal growth factor receptor (EGFR), myelin and lymphocyte protein (MAL), and breast cancer 1/2 (BRCA1/BRCA2) are independent predictors of patient survival in serous ovarian cancer [12-14]. However, the accuracy of these gene signatures remains controversial, because previous studies are merely on serous ovarian cancer without validation datasets.

Compared with other molecular detection techniques, immunohistochemistry has its own advantages: lower cost, presenting morphology for cells and tissues and less time consuming. The aim of the current study was to elucidate epithelial ovarian cancer (EOC) at the molecular level and to establish a predictive model for EOC using immunohistochemistry. To consider cancer heterogeneity and to improve prognostic accuracy, we further integrated molecular and clinicopathological factors into the risk model.

\section{Methods \\ Patients}

Our research has been approved by Ethics Committee of Ren Ji Hospital, Shanghai Jiao Tong University, School of Medicine, and informed consents were obtained from all epithelial ovarian cancer patients or their direct relatives. The tumor tissue specimens were collected from patients who were operated and confirmed with histopathology post-operation between June 2003 and December 2009 in the Department of Obstetrics and Gynaecology, Ren Ji Hospital, Shanghai, China. The subjects were divided into two groups: an experimental group $(n=161)$ and a validation group $(n=40)$. Each patient had undergone cytoreductive surgery (without neoadjuvant chemotherapy, NACT) and a standardized post-surgical course of chemotherapy based on platinum. The following factors were recorded for the experimental group $(n=161)$ : the clinicopathological characteristics (Table 1), the effect of chemotherapy based on the platinum (Table 1) and follow-up outcome. Platinum-resistant ovarian cancer (PROC) was defined as recurrence within 6 months of the completion of platinum-based chemotherapy and disease progression within 6 months during or after chemotherapy [15].

\section{Immunohistochemistry}

Using immunohistochemistry, 15 molecular markers were selected for analysis in specimens after evaluating a range of published prognostic molecular markers [11, 12, 16-22] (Additional file 1: Table S1) and considering the tumor characteristics [23]. The immunohistochemistry was performed as following: briefly, paraffin-embedded tumor specimens were antigen retrieved in a microwave at $>90{ }^{\circ} \mathrm{C}$ for $15 \mathrm{~min}$ after dewaxing and rehydration, then were blocked with $5 \%$ bovine serum albumin (BSA) for $1 \mathrm{~h}$ to reduce non-specific binding. The specimens were incubated sequentially with a rabbit anti-BRCA1 polyclonal antibody (SANTA CRUZ, Dallas, Texas, USA), a mouse anti-P53 monoclonal antibody (SANTA CRUZ, Dallas, Texas, USA), a rabbit anti-human epidermal growth factor receptor 2 (HER2) polyclonal antibody (SANTA CRUZ, Dallas, Texas, USA), a mouse anti-murine sarcoma viral oncogene homo$\log$ B (BRAF) monoclonal antibody (SANTA CRUZ, Dallas, Texas, USA), a mouse anti-Kirsten rat sarcoma viral oncogene homolog (KRAS) monoclonal antibody (MILLIPORE, Billerica, Massachusetts, USA), a rabbit anti-Ki67 polyclonal antibody (SANTA CRUZ, Dallas, Texas, USA), a rabbit anti-vascular endothelial growth factor (VEGF) polyclonal antibody (SANTA CRUZ, Dallas, Texas, USA), a rabbit anti-Notch homolog 3 (NOTCH3) polyclonal antibody (SANTA CRUZ, Dallas, Texas, USA), a rabbit anticyclin E1 (CCNE1) polyclonal antibody (SANTA CRUZ, Dallas, Texas, USA), a rabbit anti-erythroid transcription factor (GATA2) polyclonal antibody (SANTA CRUZ, Dallas, Texas, USA), a rabbit anti-forkhead box protein M1 (FOXM1) polyclonal antibody (SANTA CRUZ, Dallas, Texas, USA), a rabbit anti-BRCA2 polyclonal antibody (SANTA CRUZ, Dallas, Texas, USA), a rabbit anti-EGFR polyclonal antibody (SANTA CRUZ, Dallas, Texas, USA), a rabbit anti-phosphatase and tensin homolog (PTEN) polyclonal antibody (Zhongshan, Beijing, China), a mouse anti-multidrug resistance (MDR1) monoclonal antibody (SANTA CRUZ, Dallas, Texas, USA) for $2 \mathrm{~h}$ at 1:100 dilution and a horseradish peroxidase (HRP)-conjugated goat anti-mouse or anti-rabbit IgG antibody (Zhongshan, Beijing, China; 1:100) for 1 h. 3,3'-diaminobenzidine tetrahydrochloride (DAB; Zhongshan, Beijing, China) and hematoxylin were used to colour the slides. The semiquantitative evaluation of the staining for 15 molecular markers was performed by two pathologists in blind fashion, as described in the Additional file 1 [12, 24-32].

\section{Statistical analysis}

The outcome was the survival time from diagnosis to the date of death. The surviving patients' cut-off was the date of the last follow-up if the duration of follow-up was more than 5 years.

Multiple imputations have been advocated as an appropriate method to manage missing data [33]. The sequential regression multiple imputation (SRMI) method, serving as an imputation model, was applied to make up the missing data. We then used the multiple-imputed data sets to analyse the variables [34].

SPSS 19.0 software (IBM, Armonk, New York, USA) was used for the analyses. The differences in survival according to clinicopathological and 15 molecular factors were assessed by using a log-rank test and a univariate 
Table 1 Patient characteristics and potential prognostic factors

\begin{tabular}{|c|c|c|c|c|}
\hline Characteristics & $N$ & $(\%)$ & Median survival (months) & $p^{b}$ \\
\hline Age & & & & 0.095 \\
\hline$<40$ & 7 & 4.3 & 83.0 & \\
\hline $40-49$ & 31 & 19.3 & 33.1 & \\
\hline $50-59$ & 62 & 38.5 & 36.0 & \\
\hline $60-69$ & 33 & 20.5 & 46.0 & \\
\hline$\geq 70$ & 26 & 16.1 & 60.3 & \\
\hline Parity & & & & $0.019^{*}$ \\
\hline $0-1$ & 77 & 47.8 & 62.2 & \\
\hline $2-3$ & 66 & 41.0 & 52.0 & \\
\hline$>3$ & 15 & 9.3 & 45.0 & \\
\hline Menopause & & & & 0.716 \\
\hline Yes & 106 & 65.8 & 45.0 & \\
\hline No & 53 & 32.9 & 49.4 & \\
\hline Ascites & & & & 0.735 \\
\hline Yes & 62 & 38.5 & 37.0 & \\
\hline No & 97 & 60.2 & 59.5 & \\
\hline Peritoneal metastasis & & & & $0.004^{*}$ \\
\hline Yes & 89 & 55.3 & 31.6 & \\
\hline No & 71 & 44.1 & 60.0 & \\
\hline Lymphatic metastasis & & & & 0.905 \\
\hline Yes & 69 & 42.9 & 26.3 & \\
\hline No & 91 & 56.5 & 58.6 & \\
\hline FIGO stage & & & & $0.025^{*}$ \\
\hline 1 & 55 & 34.2 & 62.1 & \\
\hline$\|$ & 18 & 11.2 & 42.0 & \\
\hline III & 80 & 49.7 & 35.0 & \\
\hline IV & 7 & 4.3 & 18.3 & \\
\hline Histotype & & & & 0.954 \\
\hline Serous & 114 & 70.8 & 38.7 & \\
\hline Mucinous & 15 & 9.3 & 63.0 & \\
\hline Endometrioid & 13 & 8.1 & 59.0 & \\
\hline Clear cell & 9 & 5.6 & 60.4 & \\
\hline Undifferentiated & 10 & 6.2 & 29.0 & \\
\hline Grade & & & & 0.415 \\
\hline G1 & 33 & 20.5 & 61.0 & \\
\hline $\mathrm{G} 2$ & 57 & 35.4 & 55.0 & \\
\hline G3 & 69 & 42.9 & 30.1 & \\
\hline Tumor type $^{a}$ & & & & $0.003^{*}$ \\
\hline I & 57 & 35.4 & 61.0 & \\
\hline$\|$ & 101 & 62.7 & 36.0 & \\
\hline Residual disease & & & & $<0.0001^{*}$ \\
\hline$\leq 0.5 \mathrm{~cm}$ & 119 & 73.9 & 58.0 & \\
\hline$>0.5 \mathrm{~cm}$ & 40 & 24.8 & 19.4 & \\
\hline
\end{tabular}

Table 1 Patient characteristics and potential prognostic factors (Continued)

\begin{tabular}{lllll}
\hline Platinum resistance & & & & $<0.0001^{*}$ \\
Yes & 36 & 22.4 & 22.0 & \\
No & 123 & 76.4 & 58.0 & \\
\hline
\end{tabular}

"statistical significance

aased on morphological and molecular genetic analysis, EOC are divided into two categories: type I tends to be low-grade neoplasms; while type II is high-grade neoplasms [35]

${ }^{\mathrm{b}}$ Log-rank test

analysis. We established the risk model of survival via multivariate COX regression and estimated hazard rate (HR). Each risk factor's score was calculated from betacoefficient in the multivariate analysis. The risk model for every patient was the sum of the scores of each factor. The receiver-operating characteristics (ROC) curve area and the Youden index were then utilized to calculate the cut-off points of the risk model. Based on the cut-off points, the risk model of survival was divided into two classes: low risk and high risk. We then adopted an independent validation in the validation group and used the Kaplan-Meier method to analyse the difference between the two risk classes. For all analyses, $P<0.05$ was considered significant.

\section{Results}

Description of the study cohort

The clinicopathological characteristics of the experimental group $(n=161)$ were presented in Table 1 . The median survival was 48 months (range: 8-123 months); the international federation of gynaecology and obstetrics (FIGO) stage was predominantly III (49.7 \%) and I (34.2\%) at initial diagnosis; and the histopathology mainly exhibited serous epithelial ovarian cancer (70.8\%); the proportions of other pathological subtypes (mucinous, endometrioid, clear cell and undifferentiated cancer) were similar; $73.9 \%$ patients underwent the ideal tumor reductive surgery.

\section{Predictors of survival \\ Clinicopathological characteristics}

Based on the univariate analyses of clinicopathological characteristics, six variables, including parity, peritoneal metastasis, FIGO stage, tumor type [35], residual disease and platinum resistance were statistically significant risk factors for overall survival $(P \leq 0.05)$. Overall survival was more significantly associated with the tumor type than with the WHO grade (the area under the ROC curve was 0.815 vs. 0.787 ) (Fig. 1).

\section{Molecular markers}

The immunohistochemistry results of the molecular markers were presented in Additional file 1: Figure S1. 


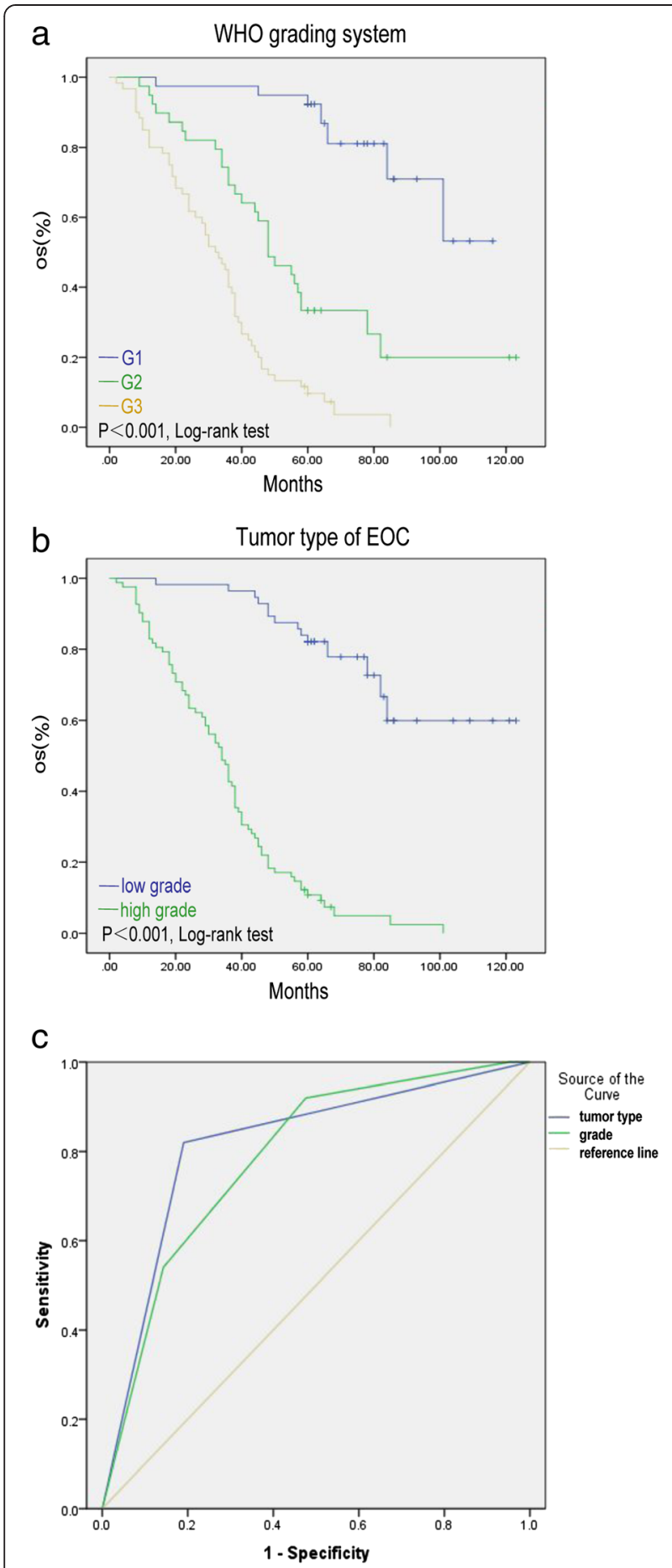

Fig. 1 Kaplan-Meier survival curve and ROC curve of WHO grading system and tumor type. a Kaplan-Meier survival curve of WHO grading system. b Kaplan-Meier survival curve of tumor type. c ROC curve of WHO grading systems and tumor type. The area was 0.815 (blue line: tumor type) vs. 0.787 (green line: WHO grade)

Using the Kaplan-Meier survival analysis, 10 molecular markers were identified to be significant correlated with overall survival (Additional file 1: Figure S2).
The relationships between the molecular markers and the clinicopathological features were illustrated in Additional file 1: Table S2. The patients with strong staining or complete absence of P53 expression, which presumably lost P53 function (Strong staining or complete absence of P53 expression is defined as TP53 mutation) tended to display increased peritoneal metastasis $(P=0.027)$ and later FIGO stage $(P=0.024)$. The tumor type was closely associated with the expression of P53 $(P=0.002)$. The strong staining or complete absence of P53 which is equivalent to loss of P53 function was most frequently observed in serous carcinomas (70.8 \%). The significant association between high HER2 expression and the tumor type $(P=0.025)$ as well as with residual disease $(P=0.002)$ was observed. Strong staining of KRAS (the KRAS mutation commonly had strong staining) in the tumor cell nuclei was significantly associated with FIGO stage $(P=0.011)$, tumor type $(P=0.025)$ and WHO grade $(P=0.027)$. The absence or weak staining of BRCA1 which was considered as BRCA1 mutation, was also significantly associated with tumor type $(P=0.048)$. The absence or weak staining of BRAF (Negative and weak staining in tumor cell cytoplasm was considered to be mutation $B R A F)(P=0.045)$ and high EGFR expression $(P=0.041)$ were associated with the ovarian cancer histotype. Serous $(69.5 \%)$ and mucinous $(11.3 \%)$ ovarian cancer had high proportions of low BRAF expression, while serous $(63.6 \%)$ and endometrioid (13.6 \%) ovarian cancer exhibited high EGFR expression. Two of the most striking findings were that (a) highly significant associations were discovered between the overexpression of HER2 (26/ 36 resistant patients, $P=0.013)$, KRAS (32/36 resistant patients, $P=0.004)$, low expression of PTEN (36/36 resistant patients, $P=0.043)$ and platinum resistance, and (b) platinum resistance $(P=0.043)$, residual disease $(P<0.001)$, the expression of VEGF $(P=0.031)$ and HER2 $(P=0.008)$, the expression of $\operatorname{BRCA} 1(P=0.05)$ and $\operatorname{KRAS}(P=0.021)$ were significantly associated with overall survival in patients with the same stages and treated with uniform therapies. There were no associations between clinicopathological characteristics and the expression of VEGF, NOTCH3, and BRCA2.

\section{The risk models}

To evaluate the association between ovarian cancer patient outcomes and the clinicopathological characteristics as well as molecular markers, we established three risk models: the clinicopathological model, the molecular model and the synthetic model comprising both clinicopathological characteristics and molecular markers. First, we performed a multivariate COX regression analysis to independently assess the relationship between clinical characteristics or molecular markers and patient survival. The results showed that parity, peritoneal metastasis, FIGO stage, tumor type and residual disease were independent prognostic factors 
among the clinical characteristics (Table 2). Among the molecular markers, HER2, KRAS, BRCA1, BRAF, and EGFR were independent and statistically significant prognostic factors for ovarian cancer patient outcomes (Table 2). The risk score of each predictor was obtained from the coefficient of the COX regression model. Consequently, the total score for the clinicopathological model was 7 points, while the total score for the molecular model was 5 points. The score for the synthetic model was generated using the sum of the scores of the above independent clinicopathological and molecular factors (12 points). The three scoring systems were shown in Table 3.

In the synthetic model, the ROC area was 0.942 with a cut-off at 6.5 points compared with the clinicopathological model (ROC area: 0.869, cut-off point: 2.5) and molecular model (ROC area: 0.884, cut-off point: 2.5) (Fig. 2a). Then we validated the three risk models for discrimination in an independent validation dataset. Likewise, the ROC area of the synthetic model (ROC area: 0.798 , cut-off point: 6.5 ) was largest than the

Table 2 The Cox regression analysis of prognostic factors in epithelial ovarian cancer

\begin{tabular}{|c|c|c|c|c|}
\hline Factors & $\beta$ & Hazard ratio & $95 \%$ confidence interval & $P$ \\
\hline \multicolumn{5}{|c|}{ Clinicopathological factors } \\
\hline Parity & & & & 0.009 \\
\hline $0-1$ & & & Reference & \\
\hline $2-3$ & 0.103 & 0.668 & $0.410-1.089$ & \\
\hline$>3$ & 1.069 & 2.911 & $1.203-7.044$ & \\
\hline Peritoneal metastasis & & & & 0.047 \\
\hline No & & & Reference & \\
\hline Yes & 1.086 & 2.963 & $1.013-8.664$ & \\
\hline FIGO stage & & & & 0.021 \\
\hline । & & & Reference & \\
\hline$\|$ & 0.722 & 2.058 & $0.603-7.025$ & \\
\hline III & 1.150 & 3.158 & $0.730-13.658$ & \\
\hline IV & 1.470 & 4.351 & $1.611-11.746$ & \\
\hline Tumor type & & & & $<0.001$ \\
\hline । & & & Reference & \\
\hline$\|$ & 1.613 & 5.017 & $2.501-10.067$ & \\
\hline Residual disease & & & & $<0.001$ \\
\hline$\leq 0.5 \mathrm{~cm}$ & & & Reference & \\
\hline$>0.5 \mathrm{~cm}$ & 1.553 & 4.725 & $2.418-9.233$ & \\
\hline \multicolumn{5}{|l|}{ Molecular factors } \\
\hline HER2 & & & & $<0.001$ \\
\hline Low expression & & & Reference & \\
\hline High expression & 1.242 & 3.463 & $1.839-6.523$ & \\
\hline KRAS & & & & $<0.001$ \\
\hline Low expression & & & Reference & \\
\hline High expression & 1.332 & 3.787 & $1.959-7.319$ & \\
\hline BRCA1 & & & & 0.003 \\
\hline High expression & & & Reference & \\
\hline Low expression & 0.957 & 2.604 & $1.398-4.849$ & \\
\hline BRAF & & & & 0.012 \\
\hline High expression & & & Reference & \\
\hline Low expression & 1.043 & 2.838 & $1.261-6.383$ & \\
\hline EGFR & & & & 0.036 \\
\hline Low expression & & & Reference & \\
\hline High expression & 0.622 & 1.862 & $1.042-3.327$ & \\
\hline
\end{tabular}


Table 3 Scoring system for the three risk models in epithelial ovarian cancer

\begin{tabular}{|c|c|c|c|c|c|c|c|c|c|c|c|c|c|}
\hline \multirow[t]{2}{*}{ Scoring } & \multicolumn{10}{|c|}{ Impact factors } & \multirow[t]{2}{*}{ Clinicopathological model } & \multirow[t]{2}{*}{ Molecular model } & \multirow{2}{*}{$\begin{array}{l}\text { Model comprising } \\
\text { clinicopathological } \\
\text { and molecular factors }\end{array}$} \\
\hline & Parity & $\begin{array}{l}\text { Peritoneal } \\
\text { metastasis }\end{array}$ & $\begin{array}{l}\text { FIGO } \\
\text { stage }\end{array}$ & Type & $\begin{array}{l}\text { Residual } \\
\text { disease }\end{array}$ & $\begin{array}{l}\text { HER2 } \\
\text { expression }\end{array}$ & $\begin{array}{l}\text { KRAS } \\
\text { expression }\end{array}$ & $\begin{array}{l}\text { BRCA1 } \\
\text { expression }\end{array}$ & $\begin{array}{l}\text { BRAF } \\
\text { expression }\end{array}$ & $\begin{array}{l}\text { EGFR } \\
\text { expression }\end{array}$ & & & \\
\hline 0 & $0-3$ & Absent & 1 & 1 & $\leq 0.5 \mathrm{~cm}$ & Low & Low & High & High & Low & & & \\
\hline 1 & $>3$ & Present & II-IV & & & High & High & Low & Low & High & & & \\
\hline 2 & & & & $\|$ & $>0.5 \mathrm{~cm}$ & & & & & & & & \\
\hline Total score & & & & & & & & & & & 7 & 5 & 12 \\
\hline
\end{tabular}



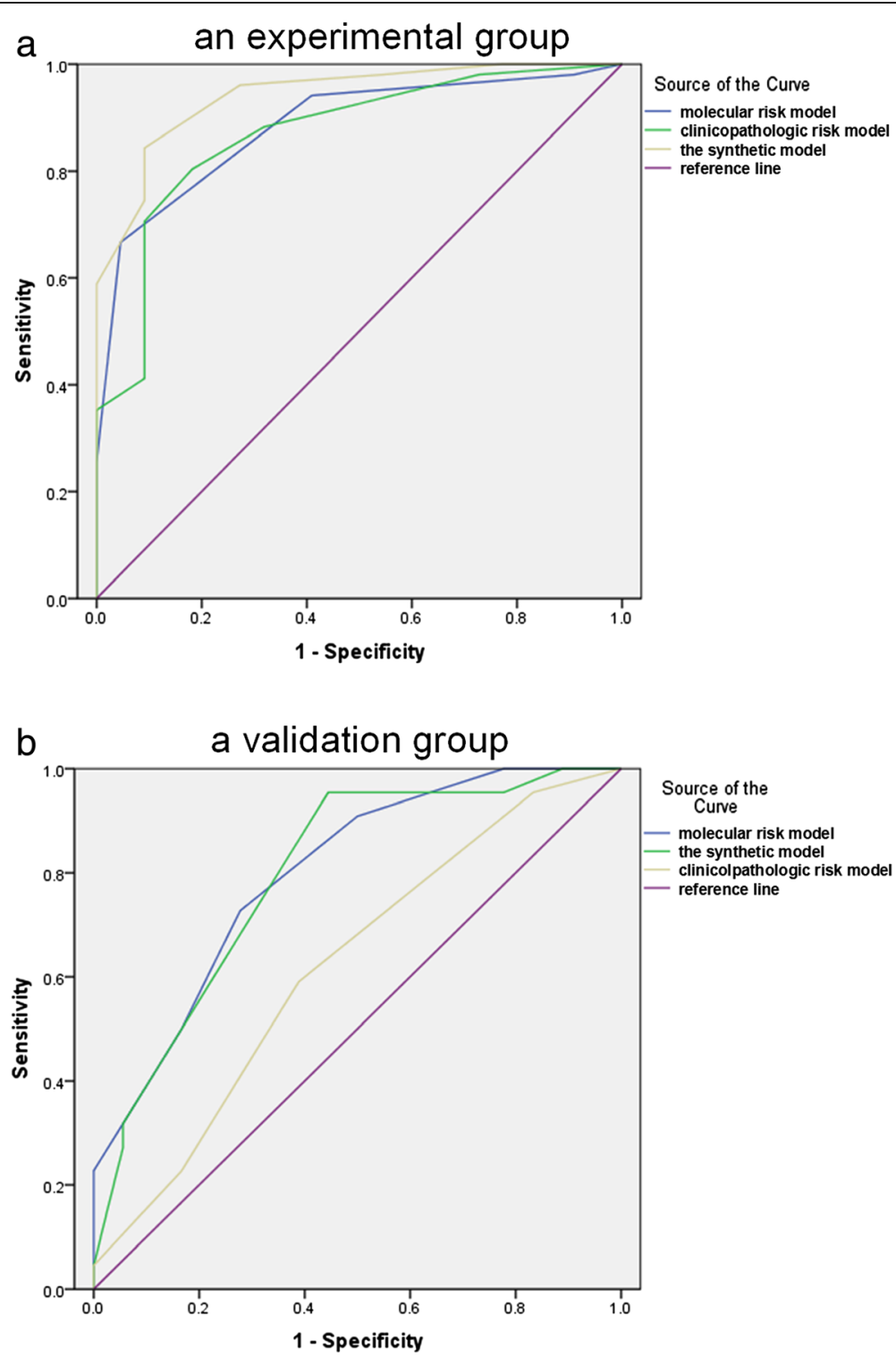

Fig. 2 ROC curves of the three risk models in the experimental group and the validation group. a In the experimental group, the areas under the curve were as following: the molecular risk model (blue line: 0.884), the clinicopathological risk model (green line: 0.869 ), and the synthetic model (yellow line: 0.942). b The ROC areas in the validation group were shown: the synthetic model (green line: 0.798), the clinicopathological model (yellow line: 0.620) and the molecular model (blue line: 0.794)

clinicopathological model (ROC area: 0.620, cut-off point: 2.5 ) and the molecular model (ROC area: 0.794, cut-off point: 2.5) (Fig. 2b). And these patients in validation group were divided into low- and high-risk classes according to the cut-off points in each model (Table 4). The differences between the two classes were significant for all three risk models (Table 4). The Kaplan-Meier survival analysis assessing the low- and high-risk classes for the three risk models was shown in Fig. 3. P value of the synthetic model was the most significant $(P<0.001)$. Moreover, the results showed that three patients with unfavorable outcomes classified as low risk by clinicopathological model were upgraded to high risk by molecular and synthetic model, and 14 patients whose survivals were close to median as high risk by clinicopathological model were downgraded to low risk by synthetic model.

\section{Discussion}

These molecular markers may be beneficial for predicting the outcome and the response to treatment for EOC, because these biomarkers can evaluate multiple genetic alterations, compared with the classical clinicopathological prognostic factors [36, 37]. To our best knowledge, although several molecular prognostic models for serous 
Table 4 Performance of the three risk models in the validation group

\begin{tabular}{|c|c|c|c|c|c|}
\hline Model & Score & No (\%) & Death (\%) & Median & $p^{c}$ \\
\hline \multirow[t]{3}{*}{ Clinicopathological model } & $0-2^{a}$ & $12(30.0)$ & $3(25.0)$ & 38.0 & \multirow{3}{*}{0.003} \\
\hline & $3-7^{\mathrm{b}}$ & 28(70.0) & 19(67.9) & 24.5 & \\
\hline & Total & 40 & $22(55.0)$ & 27.0 & \\
\hline \multirow[t]{3}{*}{ Molecular model } & $0-2^{a}$ & $20(50.0)$ & $9(45.0)$ & 30.5 & \multirow{3}{*}{0.032} \\
\hline & $3-5^{b}$ & $20(50.0)$ & $13(65.0)$ & 24.0 & \\
\hline & Total & 40 & $22(55.0)$ & 27.0 & \\
\hline \multirow{3}{*}{$\begin{array}{l}\text { Model comprising molecular } \\
\text { and clinicopathological factors }\end{array}$} & $0-6^{a}$ & $19(47.5)$ & $6(31.6)$ & 30.6 & \multirow{3}{*}{$<0.001$} \\
\hline & $7-12^{b}$ & $21(52.5)$ & $16(76.2)$ & 25.0 & \\
\hline & Total & 40 & $22(55.0)$ & 27.0 & \\
\hline
\end{tabular}

aLow-risk

${ }^{\mathrm{b}}$ High-risk

'The results were calculated using the Kaplan -Meier method

ovarian cancer have been established, there is not a clinical unified and widely applicable model. With respect to EOC, Carsten proposed the prognostic index system, which contained clinical pathological characteristic and 300 genes [38]. This prognostic system was considered to be a thorough genetic screening of EOC. In contrast, our synthetic model is a preliminary exploration to use only a handful of molecular biomarkers in conjunction with clinicopathological factors to generate a prognostic model for EOC. Notably, immunohistochemistry of these molecular markers has been now widely and routinely used for clinical diagnosis, and the uniform criteria for each molecule has been defined in immunohistopathology. In order to improve the detection efficiency and refrain from wasting EOC cancer specimen, we can prepare tissue microarray (TMA) with which all molecular markers can be tested on one slide. Even though immunohistochemistry is a semi-quantitative approach, we have validated its reliability by quantitative methods, such as flow cytometry in previous experiments [39]. Thus, the outcome of EOC patients after surgery can be conveniently and rapidly predicted using our model, and the individualized treatment regimen can be subsequently tested.

Importantly, our three risk models were validated in an independent cohort and could be applied to stratify patients into two risk classes. Nevertheless, the ROC curve area of the synthetic model was the largest. Using the cut-off point scoring system, the two risk classes in the synthetic model were more statistically significant than those in clinicopathological model or the molecular model. Moreover, the molecular model improved the accuracy of risk class classification which was previously classified in clinicopathological model. Our current data suggested that the biomarkers could be integrated in the risk model, which might lead to an improvement of clinicopathological stratification. Indeed, our synthetic risk model showed the best performance in estimating EOC patient survival.
Our risk models suggested that tumor type and residual disease were the strongest survival predictors, indicating the importance of the initial cytoreductive surgery to EOC outcome. The tumor type of EOC was defined by Shih based on morphological and molecular analysis [35]. Type I includes low-grade serous carcinoma, mucinous carcinoma, low-grade endometrioid carcinoma and clear cell carcinoma, which tend to be low-grade neoplasms with the mutations of BRAF, KRAS and PTEN; whereas type II is composed of high-grade serous carcinoma, high-grade endometrioid carcinoma and undifferentiated carcinoma which are high-grade neoplasms with high human leukocyte antigen-G (HLA-G) expression and TP53 mutation as markers. The low-grade and high-grade serous carcinoma is respectively the prototypic subtype of the two types. The University of Texas M.D. Anderson Cancer Center illuminated that lowand high-grading system of the serous ovarian cancer was associated with survival and was clinically feasible [40]. This is in line with our research that tumor type is closely associated with the overall survival, and we further revealed that the tumor type classification showed a better prognostic value than the WHO grading system. With respect to residual disease, we found that a residual tumor size $\leq 0.5 \mathrm{~cm}$ was optimal for cytoreduction, which was a predictor in the COX regression model (HR: 4.725, $P<0.001$ ), and the molecular marker HER2 was significantly associated with residual disease $(P=0.002)$. We also observed that other clinicopathological factors, including peritoneal metastasis and FIGO stage, were associated with EOC survival. These findings were consistent with Rutten's model, which is the most recognized prognostic clinicopathological model for EOC patients in recent years [41]. In addition, increased parity (especially a parity $>3$ ) was associated with high EOC risk. The parity result differed from Yang's study in which no clear association was determined between parity and ovarian cancer survival [42]. In contrast, Poule found that a 

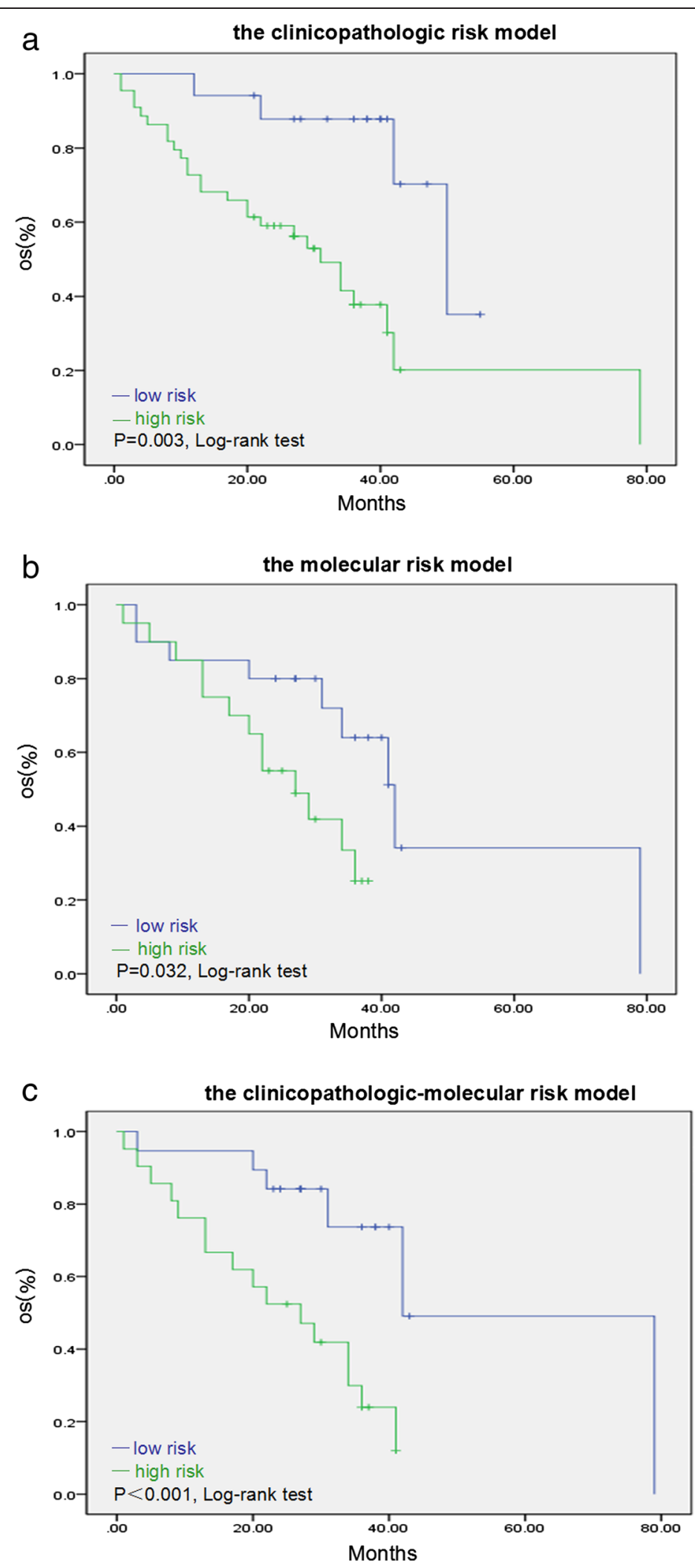

Fig. 3 The validation of the three risk models using the Kaplan-Meier survival analysis. a The clinicopathological risk model. b The molecular risk model. c The clinicopathological-molecular risk model. The blue line denotes the low-risk class and the green line denotes the high-risk class 
long-time oral-contraception was strongly correlated with less aggressive epithelial ovarian cancer due to fewer lifetime ovulatory cycles [43]. Thus, we consider that a larger sample size will be necessary to determine whether the ovulatory and multiparous increase the risk of EOC patient outcome and try to uncover the underlying mechanism.

Gene expression signatures may predict ovarian cancer outcome only in certain subtypes of ovarian cancer, including late-stage, platinum-treated and serous ovarian cancer [14, 44, 45]. Our study sought to find molecular markers to predict overall survival for all EOC types. HER2, KRAS, BRCA1, BRAF and EGFR were validated in the risk model. EGFR and HER2 are the members of the HER family, whose EGF signaling pathway has been shown to play an important role in tumor initiation, progression and metastasis. And KRAS gene can regulate the signal transduction between HER receptors and the nucleus. KRAS mutation activates KRAS protein, which continuously stimulates the EGFR activation [46]. BRAF is the downstream effector of KRAS, which was reported to be common mutation $(28-35 \%)$ in serous borderline (SB)/low grade serous ovarian cancer (LGS-OvCa) [21]. Rachel demonstrated that LGSOvCa patients with BRAF mutant were inclined to peritoneal metastasis and recurrence in the case of presence of micropapillary feature [47]. The EGFR/ HER2/KRAS/BRAF signaling pathway has been reported in pancreatic cancer and colorectal cancer other than in ovarian cancer $[48,49]$. Once mutations occur in BRAF and/or KRAS, patients are refractory to anti-EGFR therapy with poor prognosis. Skirnisdottir ever integrated EGFR into his prognostic model for early-stage EOC [12]. Our model supported notion that the four molecules in the EGFR/HER2/ KRAS/BRAF signaling pathway also produce the synergic effect, exerting their own effect on ovarian cancer progression. A new developed model of prediction of EOC should take the expression of HER2 family, KRAS and BRAF into account. TCGA analysis showed that the BRCA1 and BRCA2 mutations in $22 \%$ of the high grade serous ovarian cancer (HGS$\mathrm{OvCa}$ ) samples triggered a wide range of aberrations in DNA damage repair pathways, such as poly (ADPribose) polymerase inhibitors (PARPi) [11]. Besides the breast and ovarian cancer patients, some solid tumors such as prostate, lung, endometrial, pancreatic and colon cancer are also associated with BRCA1/2 mutations [50]. And Patch observed that the germline of mutation of BRCA1 or BRCA2 was associated with the acquired chemoresistance in HGS-OvCa [51]. In this study, our results were in line with and extend the importance of BRCA1 mutation in EOC, which was one of the critical factors of molecular markers in our synthetic prognostic model. Therefore, $B R C A$ screening is recommended to familial-risk women to prevent and diagnose EOC early. Beyond this, high HER2 expression $(P=0.013)$, high KRAS expression $(P=0.004)$ and low PTEN expression $(P=0.043)$ were associated with higher platinum chemoresistance in EOC differential from HGS-OvCa. Ovarian clear cell carcinoma (OCCC) is characterized by resistance to conventional platinum chemotherapy compared with other EOC histotypes [52]. The aberrant genes studied in OCCC included PTEN and HER2 [53]. Increased HER2 may also bind to steroid receptor coactivator 3 (SRC3), which contributes to high level of malignant cell proliferation and poor survival due to platinum resistance [54]. In addition to the above observations, Patch showed that PTEN mutation contributed to acquired chemotherapy resistance in HGS-OvCa as well [51]. Moreover, the study by Ratner indicated that EOC patients with mutated KRAS were more likely to be resistant to platinum $(\mathrm{OR}=3.18, P=0.011)$ [55]. So far, there are several pathways by which oncogenic KRAS may induce chemoresistance: first, it activates the RAF/MEK/ ERK pathway; secondly, KRAS mutation induces COX-2 expression which heightens cancer cell binding to extracellular matrix and secrets more $\mathrm{PGE}_{2}$ to facilitate cell migration and dissemination; thirdly, KRAS mutation may activate the transcription of cellular protective stress response gene nuclear factor erythroid-derived 2 (NRF2) to protect against oxidative damage and promote drug resistance [56-58]. LGS-OvCa harboring KRAS mutation is a chemoresistant disease that accounts for $10 \%$ of serous ovarian cancer. And recurrent and chemoresistant LGSOvCa patients were observed to be dramatic and durable responses to MEK inhibitor therapy [58]. Therefore, the KRAS/RAF/MEK/ERK pathway is now considered to be the key mechanism in chemoresistant LGS-OvCa. Interestingly, in our study of EOC with all histotypes, KRAS was still the cause of platinum chemoresistance. We envision that our findings may provide novel pathway for KRAS to induce chemoresistance that may ultimately lead to more targeted therapies.

There are some limitations in our analysis. Firstly, immunohistochemistry is the method to detect protein expression, which might not accurately assess the mutation of molecule. While the expression and function of TP53, KRAS, BRCA, BRAF are dependent on their genes status, they may better be detected by DNA sequencing to precisely elucidate these molecules' effect on EOC prognosis. Secondly, in order to improve the model's sensitivity and specificity, clinical multi-centre investigations are necessary to be conducted in a validationemendation-validation manner. We would attempt to solve these problems in future studies. Once resolved, the synthetic risk model may be widely applied for clinical diagnosis of EOC. 


\section{Conclusions}

Our risk model integrating clinicopathological and molecular factors was validated to predict the overall survival of EOC patients. The information obtained from the synthetic model may assist in the development of individualized and targeted therapies.

\section{Additional file}

Additional file 1: Table S1. Prognostic molecular markers in published studies. Table S2. The relationship between molecular markers and clinicopathologic characteristics. Figure S1. Single immunostaining of 15 molecular markers in epithelial ovarian cancer. Figure S2. Kaplan-Meier overall survival analyses of 15 molecular markers inepithelial ovarian cancer. (ZIP $108 \mathrm{~kb}$ )

\section{Abbreviations}

EOC: Epithelial ovarian cancer; NACT: Neoadjuvant chemotherapy; PROC: Platinum-resistant ovarian cancer; SRMI: Sequential regression multiple imputation; ROC: Receiver-operating characteristics; HGS-OvCa: High grade serous ovarian cancer; LGS-OvCa: Low grade serous ovarian cancer; OCCC: Ovarian clear cell carcinoma.

\section{Competing interests}

The authors declare that they have no competing interests.

\section{Authors' contributions}

MYZ, AMZ and WD designed the study. MYZ performed the immunohistochemical staining. XJS and YYS independently reviewed the immunostained cancer tissue sections. MYZ and GLZ drafted the manuscript. All authors read and approved the final manuscript.

\section{Acknowledgements}

We greatly appreciate Dr. Qiang Liu at the Department of Pathology of Ren Ji Hospital for providing the cancer specimens and Dr. Yong Cai at the Department of Statistics, School of Medicine, Shanghai Jiao Tong University for statistical comments. This work was supported by grant from the Science Research Foundation of the Shanghai Science and Technology Commission (12411950200).

\section{Author details}

${ }^{1}$ Department of Obstetrics and Gynecology, Ren Ji Hospital, School of Medicine, Shanghai Jiao Tong University, 160 Pujian Road, Shanghai 200127 China. ${ }^{2}$ Shanghai Key Laboratory of Gynecologic Oncology, Ren Ji Hospital, School of Medicine, Shanghai Jiao Tong University, 160 Pujian Road, Shanghai 200127, China. ${ }^{3}$ State Key Laboratory of Oncogenes and Related Genes, Renji-Med X Clinical Stem Cell Research Center, Ren Ji Hospital, School of Medicine, Shanghai Jiao Tong University, 160 Pujian Road Shanghai 200127, China. ${ }^{4}$ Department of Pathology, Ren Ji Hospital, School of Medicine, Shanghai Jiao Tong University, 160 Pujian Road, Shanghai 200127, China.

\section{Received: 16 July 2015 Accepted: 12 October 2015}

Published online: 21 October 2015

\section{References}

1. Pu X, Ye Y, Wu X. Development and validation of risk models and molecular diagnostics to permit personalized management of cancer. Cancer. 2014:120:11-9.

2. Sergeeva NS, Marshutina NV, Alentov II, Korneeva IA, Novikova EG. Serum tumor markers CA125 and HE4 in ovrian cancer patients. Vopr Onkol. 2013;59:12-21.

3. Nezhat FR, Pejovic T, Finger TN, Khalil SS. Role of minimally invasive surgery in ovarian cancer. J Minim Invasive Gynecol. 2013;20:754-65.

4. Raja FA, Counsell N, Colombo N, Pfisterer J, du Bois A, Parmar MK, et al. Platinum versus platinum-combination chemotherapy in platinum-sensitive recurrent ovarian cancer:a meta-analysis using individual patient data. Ann Oncol. 2013:24:3028-34
5. Davidson B, Tropé CG, Reich R. The clinical and diagnostic role of microRNAs in ovarian carcinoma. Gynecol Oncol. 2014;133:640-6.

6. van Houwelingen JC, ten Bokkel Huinink WW, van der Burg ME, van Oosterom AT, Neijt JP. Predictability of the survival of patients with advanced ovarian cancer. J Clin Oncol. 1989;7:769-73.

7. Hoskins P, Tu D, James K, Pater J, Koski B. Factors predictive of survival after first relapse or progression in advanced epithelial ovarian carcinoma: a prediction tree analysis-derived model with test and validation groups. Gynecol Oncol. 1998;70:224-30.

8. Clark TG, Stewart ME, Altman DG, Gabra H, Smyth JF. A prognostic model for ovarian cancer. Br J Cancer. 2001;85:944-52.

9. van Meurs HS, Schuit E, Horlings HM, van der Velden J, van Driel WJ, Mol BWJ, et al. Development and internal validation of a prognostic model to predict recurrence free survival in patients with adult granulosa cell tumors of the ovary. Gynecol Oncol. 2014;134:498-504.

10. Luborsky JL, Barua A, Shatavi SV, Kebede T, Abramowicz J, Rotmensch J. Antitumor antibodies in ovarian cancer. Am J Reprod Immunol. 2005;54:55-62.

11. The Cancer Genome Atlas Research Network, Berchuck A, Birrer M, Chien J, Cramer DW, Dao F. Integrated genomic analyses of ovarian carcinoma. Nature. 2011;474:609-15.

12. Skirnisdottir I, Seidal T, Sorbe B. A new prognostic model comprising P53, EGFR, and tumor grade in early stage epithelial ovarian carcinoma and avoiding the problem of inaccurate surgical staging. Int J Gynecol Cancer. 2004;14:259-70.

13. Berchuck A, Iversen ES, Luo J, Clarke JP, Horne H, Levine DA, et al. Microarray analysis of early stage serous ovarian cancers shows profiles predictive of favorable outcome. Clin Cancer Res. 2009:15:2448-55.

14. Verhaak RG, Tamayo P, Yang J, Hubbard D, Zhang H, Creighton CJ, et al. Prognostically relevant gene signatures of high-grade serous ovarian carcinoma. J Clin Invest. 2013:123:517-25.

15. Davis A, Tinker AV, Friedlander M. "Platinum resistant" ovarian cancer:what is it, who to treat and how to measure benefit? Gynecol Oncol. 2014;133:624-31.

16. Ahmed AA, Etemadmoghadam D, Temple J, Lynch AG, Riad M, Sharma R, et al. Driver mutations in TP53 are ubiquitous in high grade serous carcinoma of the ovary. J Pathol. 2010;221:49-56.

17. Roh MH, Yassin Y, Miron A, Mehra KK, Mehrad M, Monte NM, et al. High-grade fimbrial-ovarian carcinomas are unified by altered P53, PTEN and PAX2 expression. Mod Pathol. 2010:23:1316-24.

18. Yang D, Khan S, Sun Y, Hess K, Shmulevich I, Sood AK, et al. Association of BRCA1 and BRCA2 mutations with survival, chemotherapy sensitivity, and gene mutator phenotype in patients with ovarian cancer. JAMA. 2011;306:1557-65.

19. Vaughan S, Coward JI, Bast Jr RC, Berchuck A, Berek JS, Brenton JD, et al. Rethinking ovarian cancer:recommendations for improving outcomes. Nat Rev Cancer. 2011;11:719-25.

20. Caslini C, Capo-chichi CD, Roland IH, Nicolas E, Yeung AT, Xu X. Histone modifications silence the GATA transcription factor genes in ovarian cancer. Oncogene. 2006;25:5446-61.

21. Ardighieri L, Zeppernick F, Hannibal CG, Vang R, Cope L, Junge J, et al. Mutational analysis of BRAF and KRAS in ovarian serous borderline (atypical proliferative) tumours and associated peritoneal implants. J Pathol. 2014:232:16-22

22. Gillet JP, Calcagno AM, Varma S, Davidson B, Bunkholt Elstrand M, Ganapathi R, et al. Multidrug resistance-linked gene signature predicts overall survival of patients with primary ovarian serous carcinoma. Clin Cancer Res. 2012;18:3197-206.

23. Hanahan D, Weinberg R. Hallmarks of cancer: the next generation. Cell. 2011;144:646-68.

24. Yemelyanova A, Vang R, Kshirsagar M, Lu D, Marks MA, Shih IM, et al. Immunohistochemical staining patterns of P53 can serve as a surrogate marker for TP53 mutations in ovarian carcinoma:an immunohistochemical and nucleotide sequencing analysis. Mod Pathol. 2011;24:1248-53.

25. Wang R, Song Y, Xu X, Wu Q, Liu C. The expression of Nek7, Foxm1, and Plk1 in gallbladder cancer and their relationships to clinicopathologic features and survival. Clin Transl Oncol. 2013;15:626-32.

26. Willett RT, Greene LA. Gata2 is required for migration and differentiation of retinorecipient neurons in the superior colliculus. J Neurosci. 2011;31:4444-55.

27. Shi H, Jiang J, Ji J, Shi M, Cai Q, Chen X, et al. Anti-angiogenesis participates in antitumor effects of metronomic capecitabine on colon cancer. Cancer Lett. 2014;349:128-35.

28. Hu L, Xue F, Shao M, Deng A, Wei G. Aberrant expression of Notch3 predicts poor survival for hepatocellular carcinomas. Biosci Trends. 2013;7:152-6. 
29. De Marco C, Rinaldo N, Bruni P, Malzoni C, Zullo F, Fabiani F, et al. Multiple genetic alterations within the PI3K pathway are responsible for AKT activation in patients with ovarian carcinoma. PLoS One. 2013;8:e55362.

30. Lasota J, Kowalik A, Wasag B, Wang Z, Felisiak-Golabek A, Coates T, et al. Detection of the braf V600E mutation in colon carcinoma: critical evaluation of the imunohistochemical approach. Am J Surg Pathol. 2014;38:1235-41.

31. Kurbel S, Marjanović K, Dmitrović B. A model of immunohistochemical differences between invasive breast cancers and DCIS lesions tested on a consecutive case series of 1248 patients. Theor Biol Med Model. 2014;11. doi:10.1186/1742-4682-11-29.

32. Garg K, Levine DA, Olvera N, Dao F, Bisogna M, Secord AA, et al. BRCA1 immunohistochemistry in a molecularly characterized cohort of ovarian high-grade serous carcinomas. Am J Surg Pathol. 2013;37:138-46.

33. Graham JW. Missing data analysis: making it work in the realworld. Annu Rev Psychol. 2009;60:549-76.

34. He Y, Zaslavsky A, Landrum M, Harrington D, Catalano P. Multiple imputationin a large-scale complex survey: a practical guide. Stat Methods Med Res. 2010;19:653-70

35. leM S, Kurman RJ. Ovarian tumorigenesis: a proposed model based on morphological and molecular genetic analysis. Am J Pathol. 2004;164:1511-8.

36. Lech G, Slotwinski R, Krasnodebski IW. The role of tumor markers and biomarkers in colorectal cancer. Neoplasma. 2014;61:1-8.

37. Vollbrecht C, König K, Heukamp L, Büttner R, Odenthal M. Molecular pathology of the lungs. New perspectives by next generation sequencing. Pathologe. 2013;34:16-24.

38. Denkert C, Budczies J, Darb-Esfahani S, Györffy B, Sehouli J, Könsgen D, et al. A prognostic gene expression index in ovarian cancer-validation across different independent data sets. J Pathol. 2009;218:273-80.

39. Zhang M, He Y, Sun X, Li Q, Wang W, Zhao A, et al. A high M1/M2 ratio of tumor-associated macrophages is associated with extended survival in ovarian cancer patients. J Ovarian Res. 2014;7:19.

40. Malpica A, Deavers MT, Tornos C, Kurman RJ, Soslow R, Seidman JD, et al. Interobserver and intraobserver variability of a two-tier system for grading ovarian serous carcinoma. Am J Surg Pathol. 2007;31:1168-74.

41. Rutten MJ, Boldingh JHL, Schuit E, Trum H, van Driel W, Mol BWJ, et al. Development and internal validation of a prognostic model for survival after debulking surgery for epithelial ovarian cancer. Gynecol Oncol. 2014;135:13-8.

42. Yang L, Klint $\AA$, Lambe M, Bellocco R, Riman T, Bergfeldt K, et al. Predictors of ovarian cancer survival: a population-based prospective study in Sweden. Int J Cancer. 2008;123:672-9.

43. Poole EM, Merritt MA, Jordan SJ, Yang HP, Hankinson SE, Park Y, et al. Hormonal and reproductive risk factors for epithelial ovarian cancer by tumor aggressiveness. Cancer Epidemiol Biomarkers Prev. 2013;22:429-37.

44. Riester M, Wei W, Waldron L, Culhane AC, Trippa L, Oliva E, et al. Risk prediction for late-stage ovarian cancer by Meta-analysis of 1525 patient samples. J Natl Cancer Inst. 2014;106:dju048. doi:10.1093/jnci/dju048.

45. Sabatier R, Finetti P, Bonensea J, Jacquemier J, Adelaide J, Lambaudie E, et al. A seven-gene prognostic model for platinum-treated ovarian carcinomas. Br J Cancer. 2011;105:304-11.

46. Wang J, Yang $H$, Shen $Y$, Wang $S$, Lin D, Ma L, et al. Direct sequencing is a reliable assay with good clinical applicability for KRAS mutation testing in colorectal cancer. Cancer Biomark. 2013;13:89-97.

47. Grisham RN, lyer G, Garg K, Delair D, Hyman DM, Zhou Q, et al. Braf mutation is associated with early stage disease and improved outcome in patients with low-grade serous ovarian cancer. Cancer. 2013;119:548-54.

48. Walters DM, Lindberg JM, Adair SJ, Newhook TE, Cowan CR, Stokes JB, et al. Inhibition of the growth of patient-derived pancreatic cancer xenografts with the MEK inhibitor trametinib is augmented by combined treatment with the epidermal growth factor receptor/HER2 inhibitor lapatinib. Neoplasia. 2013;15:143-55.

49. Berg M, Soreide K. EGFR and downstream genetic alterations in KRAS/BRAF and PI3K/AKT pathways in colorectal cancer: implications for targeted therapy. Discov Med. 2012;14:207-14.

50. O'Sullivan CC, Moon DH, Kohn EC, Lee JM. Beyond breast and ovarian cancer: PARP inhibitors for BRCA mutation-associated and BRCA-like solid tumors. Front Oncol. 2014:4:42.

51. Patch A, Christie EL, Etemadmoghadam D, Garsed DW, George J, Fereday S, et al. Whole-genome characterization of chemoresistant ovarian cancer. Nature. 2015;521:489-94.
52. Tan DSP, Kaye S. Ovarian clear cell adenocarcinoma: a continuing enigma. J Clin Pathol. 2007;60:355-60.

53. Tan DSP, Miller RE, Kaye SB. New perspectives on molecular targeted therapy in ovarian clear cell carcinoma. Br J Cancer. 2013;108:1553-9.

54. Palmieri C, Gojis O, Rudraraju B, Stamp-Vincent C, Wilson D, Langdon S, et al. Expression of steroid receptor coactivator 3 in ovarian epithelial cancer is a poor prognostic factor and a marker for platinum resistance. Br J Cancer. 2013;108:2039-44.

55. Ratner ES, Keane FK, Lindner R, Tassi RA, Paranjape T, Glasgow M, et al. A KRAS variant is a biomarker of poor outcome, platinum chemotherapy resistance and a potential target for therapy in ovarian cancer. Oncogene. 2012;31:4559-66.

56. Grisham RN, Sylvester BE, Won H, McDermott G, Delair D, Ramirez R, et al. Extreme outlier analysis identifies occult mitogen-activated protein kinase pathway mutations in patients with low-grade serous ovarian cancer. J Clin Oncol. 2015. [Epub ahead of print].

57. Hoang B, Zhu L, Shi Y, Frost P, Yan H, Sharma S. Oncogenic RAS mutations in myeloma cells selectively induce cox-2 expression, which participates in enhanced adhesion to fibronectin and chemoresistance. Blood. 2006;107:4484-90.

58. Tao S, Wang S, Moghaddam SJ, Ooi A, Chapman E, Wong PK, et al. Oncogenic KRAS confers chemoresistance by upregulating NRF2. Cancer Res. 2014;74:7430-41

\section{Submit your next manuscript to BioMed Central and take full advantage of:}

- Convenient online submission

- Thorough peer review

- No space constraints or color figure charges

- Immediate publication on acceptance

- Inclusion in PubMed, CAS, Scopus and Google Scholar

- Research which is freely available for redistribution 